\section{Formation of Lateral Low Density In(Ga)As Quantum Dot Pairs in GaAs Nanoholes}

\author{
P. Alonso-González, J. Martín-Sánchez, Y. González, B. Alén, D. Fuster, and L. González* \\ Instituto de Microelectrónica de Madrid (IMM-CNM, CSIC), Isaac Newton, 8 Tres Cantos, \\ Madrid 28760 Spain
}

Received January 19, 2009; Revised Manuscript Received February 16, 2009
CRYSTAL

GROWTH

\& DESIGN

2009

VOL. 9, NO. 5

$2525-2528$

\begin{abstract}
In this work we present a growth procedure to form lateral $\operatorname{In}(\mathrm{Ga})$ As quantum dot pairs by using a low density, $2 \times 10^{8} \mathrm{~cm}^{-2}$, GaAs nanohole template previously formed in situ by droplet homoepitaxy. In particular, by changing the arsenic pressure at which InAs is grown on the template, we demonstrate the possibility to select the formation of single quantum dots (QD) or QD pairs inside each of the nanoholes. In the case of QD pairs, the ensemble photoluminescence (PL) as a function of excitation power and temperature reveals spectral signatures typical of laterally coupled nanostructures.
\end{abstract}

\section{Introduction}

The use of semiconductor quantum dots (QD) as active elements in different optoelectronic devices for quantum information technology ${ }^{1-3}$ is currently receiving much attention. Triggered sources of single photons ${ }^{3}$ or entangled photon pairs ${ }^{1}$ have been achieved using these nanostructures as active elements. In addition, by embedding them within a photonic crystal microcavity, quantum phenomena based on strong lightmatter coupling are also being studied. ${ }^{2}$ In this sense, the ability to obtain a precise control in size, spatial location, and number of nanostructures has become a highly desirable issue. ${ }^{4-6}$ In particular, the formation of different distributions of QD in close proximity ${ }^{7}$ permits a precise study of coupling and coherence that is strictly necessary for the creation of future functional units in the field of quantum computing and communication. ${ }^{8}$ In this way, the growth of vertically stacked self-assembled QD has been deeply studied during the last years allowing the direct observation of controlled coupling in a quantum dot molecule (QDM). ${ }^{9}$ For applications, however, the development of a lateral configuration of coupled nanostructures is highly desirable as it would extend the coupling to two dimensions and it would facilitate the gating technology. Experimentally, the lateral coupling of two adjacent QD has been recently demonstrated. ${ }^{10}$ In this case, the fabrication method was based on the growth selectivity of QD into previously in situ etched nanoholes. ${ }^{11}$ In a simple similar way, droplet epitaxy technique ${ }^{12,13}$ has emerged as a straightforward in situ fabrication method to obtain, in a self-assembled way, templates of low density nanoholes on GaAs substrates. In particular, by means of InAs selective growth at these nanoholes, different distributions of nanostructures ${ }^{14-18}$ have been fabricated presenting good optical properties at the single nanostructure level. ${ }^{19}$ Moreover, by using the appropriate growth conditions during the capping process of these nanostructures, the formation of mounds spatially located over the buried QD has also been demonstrated. ${ }^{2}$ This effect permits the localization of the nanostructures at the top surface, ${ }^{20}$ making easier any further technological processes for application of these nanostructures into devices.

In this work, we have explored the ability to design the formation of low density $\operatorname{In}(\mathrm{Ga})$ As QD pairs $\left(2 \times 10^{8} \mathrm{~cm}^{-2}\right)$ by the use of a template of nanoholes in situ formed by droplet

* To whom correspondence should be addressed. E-mail: palonso@ imm.cnm.csic.es. homoepitaxy on a GaAs(001) buffer layer. We have observed nucleation of single QD or QD pairs inside each of the nanoholes depending on the arsenic pressure used during InAs deposition. The photoluminescence (PL) emission of the QD pairs, in particular the evolution of the peak energy and full width at half-maximum (fwhm) as a function of excitation intensity and temperature respectively, show signatures of laterally coupled quantum systems $^{21}$ as has been previously reported. ${ }^{22,23}$

\section{Experimental Procedure}

The samples were grown by molecular beam epitaxy (MBE) and topographically characterized by atomic force microscopy (AFM), Nanotec equipment, using a Nanosensors Si tip with a force constant of $40 \mathrm{~N} / \mathrm{m}$ and a nominal radius of curvature $<10 \mathrm{~nm}$. The emission properties of the obtained nanostructures were measured at $T=30 \mathrm{~K}$ with a standard photoluminescence (PL) setup using a frequencydoubled Nd:YAG laser as excitation source $(\lambda \operatorname{exc}=532 \mathrm{~nm}$, spot diameter $\approx 200 \mu \mathrm{m}$ ).

The experimental procedure starts growing a $0.5 \mu \mathrm{m}$ thick undoped GaAs buffer layer at a growth rate $\mathrm{rg}=0.5$ monolayers per second $(\mathrm{ML} / \mathrm{s}), \mathrm{As}_{4}$ beam equivalent pressure (BEP) of $2 \times 10^{-6}$ Torr, and substrate temperature $T_{\mathrm{s}}=580^{\circ} \mathrm{C}$ on GaAs (001) substrates. The rootmean-square (rms) roughness of this surface is typically $0.24 \mathrm{~nm} . T_{\mathrm{s}}$ is then decreased to $500{ }^{\circ} \mathrm{C}$, and the nanohole template formation process is performed. It consists of a two-step process where metallic $\mathrm{Ga}$ droplets are first formed on the surface to be finally exposed to an As atmosphere. In particular, the growth protocol followed for the formation of Ga droplets consists of the opening of the Ga shutter during $20 \mathrm{~s}$, with the cell providing a flux equivalent to the growth of GaAs at $0.5 \mathrm{ML} / \mathrm{s}$. Simultaneously, the As cell is pulsed in cycles of $0.2 \mathrm{~s}$ open $/ 0.8 \mathrm{~s}$ closed at a BEPAs $_{4}=5 \times 10^{-7}$ Torr. The result of this process is the formation of $\mathrm{Ga}$ droplets spread all over the surface with a density of $2 \times 10^{8} \mathrm{~cm}^{-2}$. Finally, these Ga droplets are annealed under As atmosphere during $6 \mathrm{~min}$ at $\mathrm{BEP}_{\mathrm{As} 4}=5 \times 10^{-7}$ Torr. About $30 \mathrm{~s}$ before the completion of the 6 min annealing period, the arsenic cell cracker is operated. This process ensures the production of an $\mathrm{As}_{2}$ beam at the onset of InAs deposition. During this time, the substrate temperature is also increased to $510{ }^{\circ} \mathrm{C}$. Before any InAs deposition, we observe that the surface presents randomly located nanoholes with an elongated structure along [110] direction and a density of $2 \times 10^{-8}$ $\mathrm{cm}^{-2}$. Their dimensions are $h=4.4 \pm 0.7 \mathrm{~nm}$ for the nanoholes depth and $\mathrm{D}_{1}=43 \pm 3 \mathrm{~nm}$ and $\mathrm{D}_{2}=95 \pm 5 \mathrm{~nm}$ for the nanoholes mean diameter along [1-10] and [110] directions, respectively. On this kind of patterned surface, 1.5 ML of InAs are deposited using two different $\mathrm{As}_{2}$ pressures while keeping the rest of growth parameters constant. BEP $\mathrm{As}_{2}$ used was $3.5 \times 10^{-7}$ Torr and $1 \times 10^{-6}$ Torr. InAs was deposited following a growth sequence consisting of 0.1 monolayers (ML) at a growth rate of $0.05 \mathrm{ML} / \mathrm{s}$ followed by a pause of $2 \mathrm{~s}$ under the used $\mathrm{As}_{2}$ flux. The substrate temperature is maintained during the 


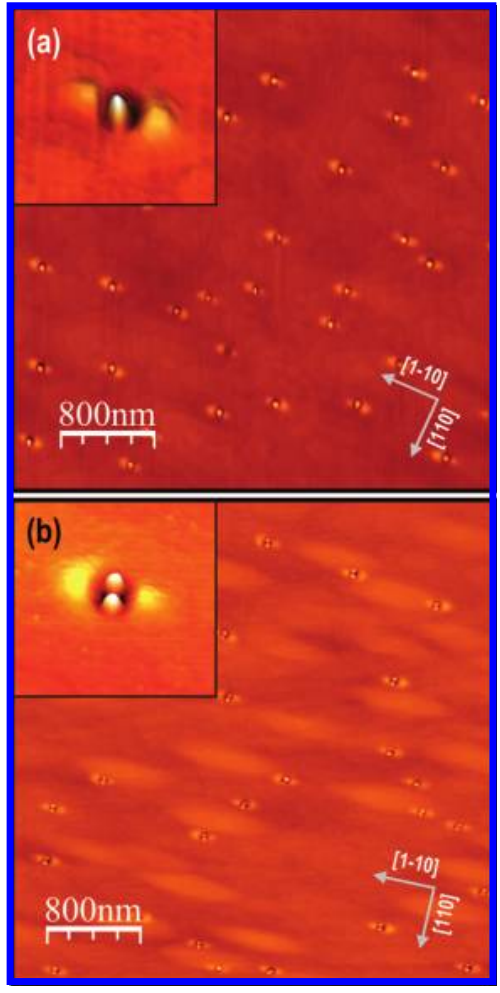

Figure 1. (a) AFM image $(4 \mu \mathrm{m} \times 4 \mu \mathrm{m})$ showing single quantum dots (QD) into GaAs nanoholes after growing 1.5 ML of InAs at $\mathrm{BEPAs}_{2}=3.5 \times 10^{-7}$ Torr. The inset shows a detailed 3D image of a single QD. (b) AFM image $(4 \mu \mathrm{m} \times 4 \mu \mathrm{m})$ showing QD pairs formed into GaAs nanoholes after growing $1.5 \mathrm{ML}$ of InAs at BEPAs $s_{2}=1 \times$ $10^{-6}$ Torr. The inset shows a detailed 3D image of a QD pair.

InAs deposition process at $510{ }^{\circ} \mathrm{C}$. A $(2 \times 4)$ surface reconstruction was always observed during InAs deposition at the used $\mathrm{As}_{2}$ pressures.

Once the InAs is deposited, a $100 \mathrm{~nm}$ thick capping layer is grown. It consists of an initial $20 \mathrm{~nm} \mathrm{GaAs}$ layer grown at $T_{\mathrm{s}}=510^{\circ} \mathrm{C}$ under $\mathrm{As}_{2}$ at $\mathrm{BEPAs}_{2}=1 \times 10^{-6}$ Torr and a final $80 \mathrm{~nm} \mathrm{GaAs}$ layer at conventional MBE GaAs growth conditions, $T_{\mathrm{s}}=580^{\circ} \mathrm{C}$ and BEPAs $4=$ $2 \times 10^{-6}$ Torr. ${ }^{16}$ Finally, on the top surface, the process for obtaining uncapped nanostructures on the nanoholes template is repeated for AFM topographic measurements.

\section{Results and Discussion}

Figure 1 shows AFM images corresponding to the deposition of 1.5 ML of InAs into the formed GaAs template at the two different $\mathrm{As}_{2}$ pressures used in this work. Figure 1a shows the nucleation result when the lower As $\mathrm{s}_{2}$ pressure $\left(\mathrm{BEPAs}_{2}=3.5\right.$ $\times 10^{-7}$ Torr) is used during InAs deposition. It can be observed that a single QD is nucleated inside each of the nanoholes. The single QD occupancy is around $95 \%$. In the inset of this figure a $0.5 \mu \mathrm{m} \times 0.5 \mu \mathrm{m}$ 3D AFM image shows in detail one of these single QD. A different InAs nucleation behavior is observed in Figure 1b; in this case, by using the higher $\mathrm{As}_{2}$ pressure $\left(\mathrm{BEPAs}_{2}=1 \times 10^{-6}\right.$ Torr) during InAs deposition, QD pairs aligned along [110] direction are formed inside each of the GaAs nanoholes. The double QD occupancy for this case is around $98 \%$ indicating a highly selective nucleation process at the nanoholes. The inset of this figure shows one of the QD pairs in a $0.5 \mu \mathrm{m} \times 0.5 \mu \mathrm{m}$ 3D AFM image.

Figures $2(\mathrm{a}-\mathrm{c})$ shows detailed $0.25 \mu \mathrm{m} \times 0.25 \mu \mathrm{m}$ 3D AFM images for the initial nanohole, single QD, and QD pair structures, respectively. At their right, corresponding profiles along the [110] direction are also shown. The nanoholes, as already commented, are elongated structures along [110] direc-

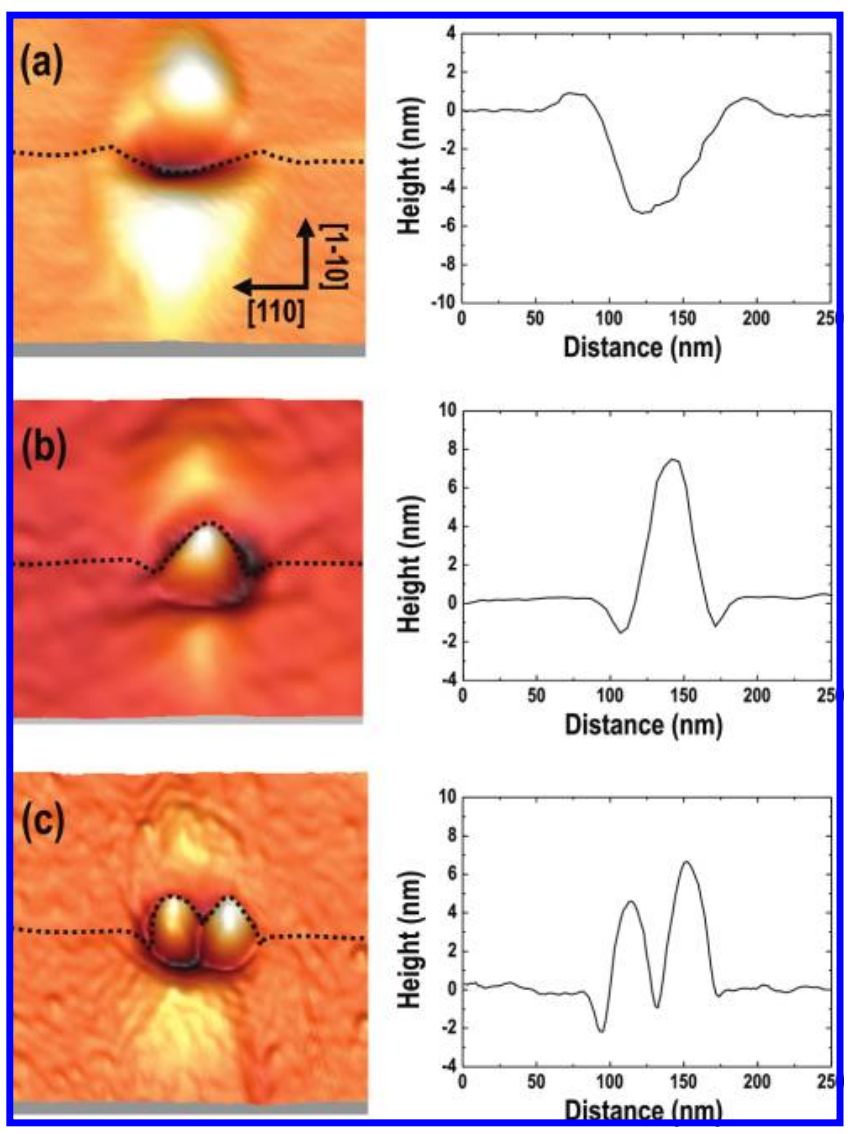

Figure 2. 3D AFM images $(0.25 \mu \mathrm{m} \times 0.25 \mu \mathrm{m})$ corresponding to: (a) the initial GaAs nanohole, (b) single quantum dot occupancy at the nanohole, and (c) QD pair formation. Respective profiles of the nanoholes, single QD, and QD pairs are shown on the right.

tion with dimensions around 95nm in diameter along the [110] direction and $4.4 \pm 0.7 \mathrm{~nm}$ in depth (Figure 2a). It has to be noticed that experiments reproducing the same growth conditions for the InAs nanostructures formation but without opening the In cell were performed and no significant differences in the dimensions of the nanoholes were observed.

Although AFM tip convolution effects at these small holes impede any possible facet identification inside them, it is expected, according to their orientation, that the lateral walls would be mainly B-type facets with steps along [110] direction.

In the case of single QD at the nanoholes (Figure 2b), these nanostructures show mean diameter of $58 \pm 3 \mathrm{~nm}$ and height of $7.4 \pm 0.8 \mathrm{~nm}$, both quantities referred to the flat surface plane. In the case of QD pairs formation, systematic differences for QD heights are observed into the pairs, this effect permits to calculate two different height distributions, as represented by a two peaks Gaussian fit in Figure 3, with average values of 5.3 $\mathrm{nm} \pm 17 \%$ and $6.6 \mathrm{~nm} \pm 24 \%$. It can be also observed in the QD pairs that the separation edge to edge between adjacent QD is practically zero within AFM resolution. A statistical mean value of $37 \pm 4 \mathrm{~nm}$ is obtained for their diameter and peak to peak separation. According to previous reports on QD molecules $^{11}$ this QD peak interdistance is short enough to potentially observe quantum coupling phenomena. We want to notice that, as clearly observed in Figure $2 b$, single QD at the nanoholes are larger in size than those forming the pairs. However, we have observed that this size effect does not lead to a different PL peak energy (Figure 4). A similar QD PL energy emission in spite of size differences could be ascribed to a different $\mathrm{In}(\mathrm{Ga})$ As composition in the single QD and QD pairs. This 


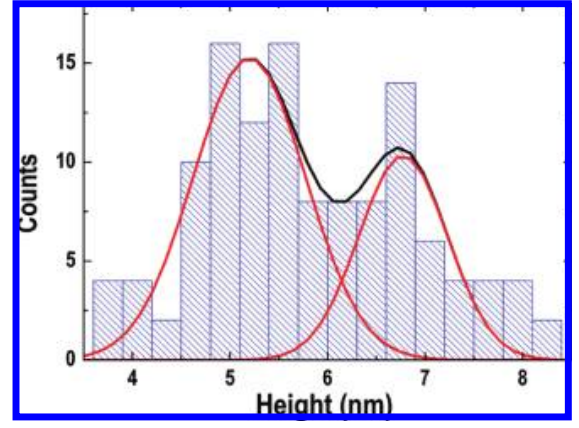

Figure 3. Distribution of QD heights in the QD pair sample.

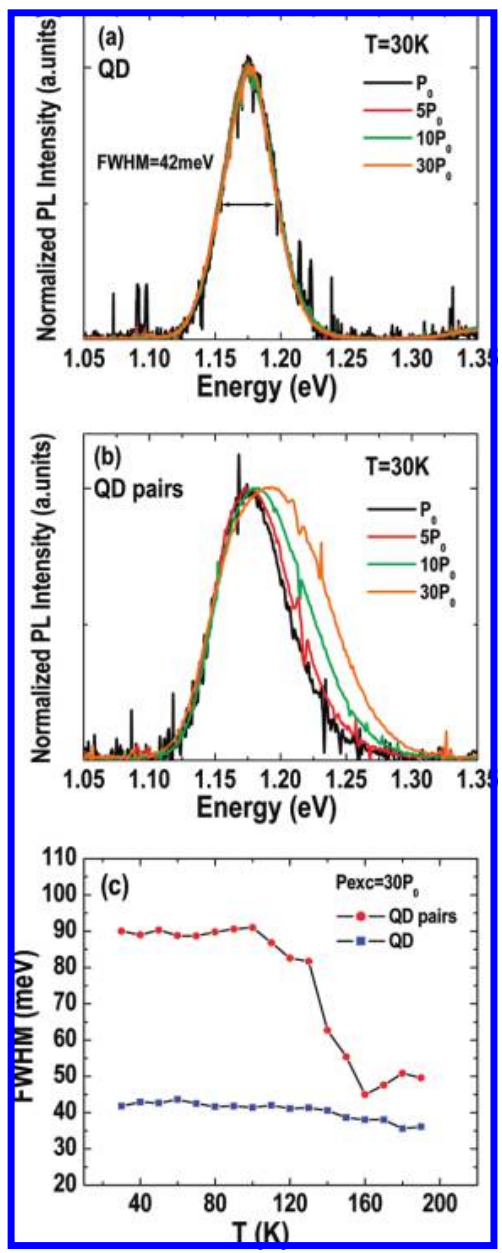

Figure 4. (a) Normalized single QD sample photoluminescence (PL) as a function of the excitation power. (b) Same for the QD pairs sample. (c) PL fwhm evolution as a function of temperature for QD pairs (red circles) and single QD (blue squares).

effect would be explained by a different Ga and In filling ratio at the nanoholes for the two different $\mathrm{As}_{2}$ pressures used in this work. Thus, it seems that the role of arsenic pressure on the nucleation of either one or two QD inside otherwise identical nanoholes would be a change of Ga/In intermixing. According to previous works, ${ }^{5,24}$ the change of reactivity of the different facets with arsenic pressure causes changes in the incorporation rates of the element III atoms. This effect would lead to the formation of QD with different composition and equilibrium size. The final result is then that for the same nanoholes profile, one QD (larger size, higher Ga content) or two QD (smaller size, lower Ga content) are nucleated at the nanoholes.
The normalized ground-state PL from the QD and the QD pairs as a function of the excitation power is shown in Figure $4 \mathrm{a}$ and $\mathrm{b}$, respectively. The low excitation power used in these experiments $\left(P_{0}=0.03 \mathrm{~mW}\right)$ avoids any emission from excited states as seen by the linear dependence of their integrated PL intensity (not shown). ${ }^{22}$ The single QD sample luminescence, shown in Figure 4a, is characterized by a symmetric peak at $1.175 \mathrm{eV}$ with a fwhm of $42 \mathrm{meV}$. Meanwhile, for paired nanostructures, shown in Figure 4b, the emission is centered at $1.18 \mathrm{eV}$ with a broader fwhm of $63 \mathrm{meV}$. The similar PL energy observed for both kind of nanostructures independently of their distinct average size can be explained by their different $\operatorname{In}(\mathrm{Ga}) \mathrm{As}$ composition as commented above. In the single QD sample, a larger Ga content is necessary to push their energy levels upward and compensate their bigger average height. Increasing the excitation power, the luminescence of the QD pairs becomes asymmetric toward higher energies. In the same excitation range, no excited-state emission could be detected in the single QD sample which otherwise had a smaller number of QD per unit area. This fact has been related recently with the internal redistribution of carriers between the small and the large QD of each QD pair implying a certain degree of lateral coupling. ${ }^{22,23}$ The participation of other resonant processes different from quantum tunneling in the ground state, as recently investigated by micro-PL studies of QD molecules, can not be concluded from our data. ${ }^{11}$

We observe also marked differences among the single QD and the QD pair samples investigating the temperature dependence of the PL fwhm shown in Figure 3c. Increasing the temperature, the single QD sample emission bandwidth remains almost constant in the studied range. Meanwhile, for the QD pairs, above $90 \mathrm{~K}$, the fwhm diminishes rapidly from its low temperature value $(90 \mathrm{meV})$ up to $\sim 50 \mathrm{meV}$ at $200 \mathrm{~K}$. It is well-known that thermally activated carrier redistribution from the QD toward the wetting layer can produce a reduction of the fwhm as the temperature is increased. ${ }^{25}$ Given the shallower confinement of nanostructures emitting at $1.25 \mathrm{eV}$ compared with typical QD, these phenomena can not be completely neglected in our case even at moderate temperatures. According to previous studies in similar systems, the differences found between both samples could also be related to carrier tunneling involving charge redistribution between the QD in the pairs. ${ }^{22,23}$

These PL results related to emission line shape dependence with excitation power and fwhm evolution with $\mathrm{T}$ can then be considered as preliminar signatures of lateral coupling in the QD pairs. However, to this respect, further optical studies at the individual QD pair level are mandatory to firmly establish the existence of QD molecules in this system.

\section{Conclusions}

In summary, we have studied the formation of low density $\operatorname{In}(\mathrm{Ga})$ As QD pairs laterally aligned along the [110] crystal direction. We have observed that the As pressure used during InAs deposition is a key parameter in order to control the formation of either single QD or QD pairs into previously in situ formed nanoholes. In particular, under the appropriate conditions we can obtain a $98 \%$ of double occupancy at the nanoholes. Our study of the ensemble emission of these QD pairs reveals spectral signatures usually associated to laterally coupled nanostructures which need to be confirmed by further investigation of their individual emission properties.

Acknowledgment. We gratefully acknowledge the financial support by the Spanish MICINN (TEC2008-06756-C03-01, 
Consolider-QOIT CSD2006-0019), CAM (S-505/ESP/000200) and by the European Commission through SANDIE Network of Excellence (No. NMP4-CT-2004-500101). P.A.G. thanks the I3P program.

\section{References}

(1) Stevenson, R. M.; Young, R. J.; Atkinson, P.; Cooper, K.; Ritchie, D. A.; Shields, A. J. Nature (London) 2006, 439, 179.

(2) Hennessy, K.; Badolato, A.; Wigner, M.; Gerace, D.; Atatüre, M.; Gulde, S.; Fält, D.; Hu, E. L.; Imamoglu, A. Nature (London) 2007, $445,896$.

(3) Intallura, P. M.; Ward, M. B.; Karimov, O. Z.; Yuan, Z. L.; See, P.; Shields, A. J.; Atkinson, P.; Ritchie, D. A. Appl. Phvs. Lett. 2007, 91, 161103.

(4) Heidemeyer, H.; Müller, C.; Schmidt, O. G. J. Cryst. Growth 2004, $261,444$.

(5) Songmuang, R.; Kiravittaya, S.; Schmidt, O. G. Appl. Phvs. Lett. 2003, 82, 2892.

(6) Atkinson, P.; Ward, M. B.; Bremner, S. P.; Anderson, D.; Farrow, T.; Jones, G. A. C.; Shields, A. J.; Ritchie, D. A. Jpn. J. Appl. Phvs. 2006, 45, 2519

(7) Li, S. S.; Xia, J. B.; Yuan, Z. L.; Xu, Z. Y.; Ge, W.; Wang, X. R.; Wang, Y.; Wang, J.; Chang, L. L. Phvs. Rev. B 1996, 54, 11575.

(8) Robledo, L.; Elzerman, J.; Jundt, G.; Atatüre, M.; Högele, A.; Fält, S.; Imamoglu, A. Science 2008, 320, 772.

(9) Stinaff, E. A.; Scheibner, M.; Bracker, A. S.; Ponomarev, I. V.; Korenev, V. L.; Ware, M. E.; Doty, M. F.; Reinecke, T. L.; Gammon, D. Science 2006, 311, 636.

(10) Beirne, G. J.; Hermannstädter, C.; Wang, L.; Rastelli, A.; Schmidt, O. G.; Michler, P. Phvs. Rev. Lett. 2006, 96, 137401.
(11) Wang, L.; Rastelli, A.; Kiravittaya, S.; Atkinson, P.; Ding, F.; Bufon, C. C. B.; Hermannstädter, C.; Witzany, M.; Beirne, G. J.; Michler, P.; Schmidt, O. G. N. J. Phvs. 2008, 10, 045010.

(12) Mano, T.; Watanabe, K.; Tsukamoto, S.; Fujioka, H.; Oshima, M.; Koguchi, N. J. Crvst. Growth 2000, 209, 504.

(13) Lee, J. H.; Wang, Zh. M.; Ware, M. E.; Wijesundara, K. C.; Garrido, M.; Stinaff, E. A.; Salamo, G. J. Crust. Growth Des. 2008, 8, 19451951.

(14) Lee, J. H.; Wang, Zh. M.; Strom, N. W.; Mazur, Yu. I.; Salamo, G. J. Appl. Phvs. Lett. 2006, 89, 202101.

(15) Wang, Zh. M.; Liang, B. L.; Sablon, K. A.; Salamo, G. J. Appl. Phvs. Lett. 2007, 90, 113120.

(16) Alonso-González, P.; Fuster, D.; González, L.; Martín-Sánchez, J.; González, Y. Appl. Phvs. Lett. 2008, 93, 183106.

(17) Liang, B. L.; Wang, Zh. M.; Wang, X. Y.; Lee, J. H.; Mazur, Y. I.; Shih, C. K.; Salamo, G. J. ACS Nano 2008, 2, 2219.

(18) Strom, N. W.; Wang, Zh. M.; Lee, J. H.; Abuwaar, Z. Y.; Mazur, Yu. I.; Salamo, G. J. Nan. Res. Lett. 2007, 2, 112.

(19) Alonso-González, P.; Alén, B.; Fuster, D.; González, Y.; González, L.; Martínez-Pastor, J. Appl. Phvs. Lett. 2007, 91, 163104.

(20) Alonso-González, P.; González, L.; Fuster, D.; González, Y.; Taboada, A. G.; Ripalda, J. M.; Beltrán, A. M.; Sales, D. L.; Ben, T.; Molina, S. I. Cryst. Growth Des. 2009, 9 (2), 1216.

(21) Li, S. S.; Xia, J. B. Appl. Phvs. Lett. 2007, 91, 092119.

(22) Liang, B. L.; Wong, P. S.; Nuntawong, N.; Albrecht, A. R.; Tatebayashi, J.; Rotter, T. J.; Balakrishnan, G.; Huffaker, D. L. Appl. Phvs. Lett. 2007, 91, 243106.

(23) Lippen, T. v.; Silov, A. Yu.; Nötzel, R. Phvs. Rev. B 2007, 75, 115414.

(24) Shen, X. Q.; Kishimoto, D.; Nishinaga, T. Jpn. J. Appl. Phvs. 1994, $33,11$.

(25) Sanguinetti, S.; Henini, M.; Grassi, M.; Capizzi, M.; Frigeri, P.; Franchi, S. Phvs. Rev. B 1999, 60, 8276.

CG900065V 